\title{
Agrupamento de espécies arbóreas de uma floresta tropical por características de solo(1)
}

\author{
Jorge Araújo de Sousa Lima( ${ }^{(2)}$, Neli do Amaral Meneguelli(2), Aderaldo Batista Gazel Filho(3) e Daniel Vidal Pérez(2)
}

\begin{abstract}
Resumo - A competição por nutrientes é um dos principais fatores que regulam tamanho e distribuição das populações arbóreas nos ecossistemas florestais da Amazônia, dada sua escassez na maioria dos solos da região. O objetivo deste trabalho foi agrupar parte das espécies arbóreas de uma floresta, por meio das características do solo. Foram utilizados dados de 32 espécies mais abundantes, distribuídas em 240 subparcelas de 10x10 m, localizadas em 12 parcelas de 1 ha, aleatoriamente demarcadas em uma floresta primária do Estado do Amapá, Amazônia Oriental. De acordo com técnicas de análises multivariadas, separaram-se as espécies em três grupos, que ocuparam diferentes faixas de variáveis químicas e texturais de solo. As variáveis de solo mais importantes na separação dos grupos foram $\mathrm{Ca}$, $\mathrm{Mg}, \mathrm{K}$ e Al. As espécies da família Melastomataceae concentraram suas populações em condições relacionadas a indicadores de menor fertilidade do solo. Os resultados sugerem que o substrato exerce papel importante no tamanho e na distribuição das populações arbóreas na floresta primária estudada.

Termos para indexação: Amazônia, estabelecimento da planta, população vegetal, análise multivariada, latossolo.
\end{abstract}

\section{Grouping tree species of a tropical forest based on soil characteristics}

\begin{abstract}
Among the factors that regulate size and distribution of tree populations in many forest ecosystems of Amazonia, competition for soil nutrients can be mentioned as one of the most important, due to its scarcity in most of the soils of that region. The objective of this work was to group part of the arboreal species of the tropical forest based on soil characteristics. The data of 32 more abundant species of a primary forest of Amapá State, Eastern Amazonia, distributed in 240 subplots of $10 \times 10 \mathrm{~m}$, located in 12 plots of $1 \mathrm{ha}$, randomly demarcated was used. According to cluster and canonical discriminate analysis, species were grouped into three clusters that represent ranges of chemical and textural variables of soil. The most important variables for contrasting of groups were $\mathrm{Ca}, \mathrm{Mg}, \mathrm{K}$ and Al. The species of Melastomataceae, concentrated their populations along conditions related to lower soil fertility. Results suggest that the substrate plays an important role on size and distribution of tree populations at the primary forest studied.
\end{abstract}

Index terms: Amazonia, plant establishment, plant population, multivariate analysis, oxisol.

\section{Introdução}

A floresta tropical é um dos ambientes naturais mais complexos da Terra, sendo sua composição e estrutura determinadas, principalmente, pelo clima,

\footnotetext{
(1) Aceito para publicação em 24 de setembro de 2002.

(2) Embrapa-Centro Nacional de Pesquisa de Solos, Rio de Janeiro, Rua Jardim Botânico, 1024, CEP 22460-000 Rio de Janeiro, RJ. E-mail: jorge@cnps.embrapa.br, neli@cnps.embrapa.br, daniel@cnps.embrapa.br

(3) Embrapa-Centro de Pesquisa Agroflorestal do Amapá, Caixa Postal 10, CEP 68906-670 Macapá, AP. E-mail: aderaldo@cpafap.embrapa.br
}

solo, estado sucessional da vegetação e a história natural de cada sítio (Huston, 1980; Jordan \& Herrera, 1981; Lamprecht, 1986; Whitmore, 1990; Megger et al., 1994). A proporção da importância de cada fator torna-se difícil de ser avaliada porque é, praticamente, impossível isolar os demais fatores presentes na intrincada malha de interações entre árvores e ambiente.

Um aspecto importante da ecologia florestal é a densidade das populações arbóreas, o que inclui espécies de importância econômica. Entre os fatores determinísticos que regulam a presença e o tamanho dessas populações, podem ser citados: as interações inter e intra-específicas que se expressam, por exem- 
plo, por meio de mecanismos denso-dependentes, ainda não claramente compreendidos (Harms et al., 2000); a ação de dispersores, de predadores de sementes e plântulas (Condit et al., 1996; Connel \& Green, 2000) e a competição por diversos recursos (Begon et al., 1986), como água, luz e nutrientes, este último fator especialmente limitado na maioria dos solos da Amazônia (Rodrigues, 1996).

Eventos aleatórios influenciam sobremaneira a densidade e a distribuição das espécies em ambientes naturais. Rebele (2000) aponta diversos meios pelos quais a ação de fatores determinísticos é alterada como justificativa para a coexistência da expressiva quantidade de espécies nos ambientes naturais. Brokaw \& Busing (2000) indicam que embora a distribuição da luz seja um importante gradiente no ambiente florestal, há poucas evidências de que sua importância na composição e estrutura das comunidades arbóreas que se formam nas clareiras seja maior do que o acaso. Há, entretanto, a escassez de recursos que determina a competição (Begon et al., 1986), influenciando, evidentemente, as interações ecológicas da floresta tropical e, por conseguinte, a distribuição e densidade das espécies.

A capacidade de subsistir em solos ácidos, com alta diluição de nutrientes e alta saturação por alumínio, é fator ambiental determinante na Amazônia, pois caracteriza a maioria dos solos da região (Rodrigues, 1996). Tal condição sugere que o conceito agronômico de baixa fertilidade natural, que é útil para sistemas agrícolas, não compõe o modelo ambiental que ajude a explicar a exuberância em formas de vida e biomassa, da floresta tropical. Nesse ambiente, atuam mecanismos diferenciados de economia de nutrientes entre as espécies (Jordan \& Herrera, 1981), o considerável efeito da constante deposição e decomposição de detritos orgânicos sobre o solo, a respiração de raízes e microorganismos do solo (Singh et al., 1989; Markewitz et al., 2001), além da capacidade de realizar associações simbióticas, principalmente com micorrizas (Siqueira et al., 1998), com evidências de que o P seja o principal nutriente a limitar a produção de serapilheira em florestas tropicais (Vitousek, 1984).

Jordan \& Herrera (1981) caracterizam como estratégia oligotrófica a adaptação a ecossistemas pobres em nutrientes. Jordan (1991) indica a maior pro- dução de raízes, em relação à parte aérea, como mecanismo para assegurar a nutrição por meio da exploração de um maior volume de solo, ao passo que Sternberg et al. (1998), nessas condições, demonstram que mesmo plantas jovens podem desenvolver raízes de $4 \mathrm{~m}$ de profundidade. Outro recurso importante, demonstrado por Vitousek \& Sanford Junior (1986), é a diminuição no teor foliar de nutrientes de certas espécies quando subsistentes em solos mais pobres. Outros autores indicam, ainda, a capacidade de algumas espécies em modificar o solo na região de suas rizosferas (Olson et al., 1981).

O objetivo deste trabalho foi agrupar parte das espécies arbóreas de uma floresta por meio das características do solo.

\section{Material e Métodos}

O recurso estudado é uma fração de 100 ha $(1.000 x$ $1.000 \mathrm{~m}$ ) de floresta primária de terra firme, que corresponde à Floresta Ombrófila Densa das Terras Baixas (Veloso et al., 1991; IBGE, 1992), demarcada numa propriedade de 1.000 ha, pertencente à Embrapa-Centro de Pesquisa Agroflorestal do Amapá, Município de Mazagão, AP, ramal do Camaipi, denominado Floresta do Camaipi, localizada pelas coordenadas $0^{\circ} 10^{\prime} \mathrm{N}$ e 513' W. O clima, segundo classificação de Köppen, é do tipo Ami, com totais pluviométricos anuais próximos a $2.000 \mathrm{~mm}$. $\mathrm{O}$ período chuvoso concentra-se entre os meses de janeiro a julho.

A topografia é suavemente ondulada e o solo está classificado como Latossolo Amarelo distrófico típico (Tabela 1), (Embrapa, 1999), tendo-se desenvolvido sobre sedimentos areno-argilosos, capeados por microconglomerado de matriz argilosa do Terciário, com intrusões de planície fluvial (Rabelo, 1999).

Estabeleceram-se 12 parcelas quadradas de 1 ha, distribuídas aleatoriamente e afastadas entre si, em pelo menos $50 \mathrm{~m}$. Cada parcela foi subdividida em 100 subparcelas de $100 \mathrm{~m}^{2}$ (10x10 m) (Synnott, 1979). Em cada uma delas, amostrou-se o solo, escolhendo-se, ao acaso, 20 subparcelas. Nestas, foram identificadas no campo ou em laboratório, com amostras dendrológicas, todas as árvores, pelo menos até a família botânica, com diâmetro à altura do peito $(\mathrm{DAP}=1,30 \mathrm{~m})$ a partir de $5 \mathrm{~cm}$. As amostras de solo constituíram-se de três tradagens, à profundidade de $0-20 \mathrm{~cm}$.

Na definição das espécies foram estabelecidos arbitrariamente os seguintes critérios, levando em conta, a exemplo de Condit et al. (1996), em estudos biométricos em 
florestas tropicais, o rigor estatístico: (1) consideraram-se somente as espécies presentes nas 240 subparcelas amostradas para análise de solo; (2) que as espécies deveriam estar presentes em, pelo menos, quatro parcelas e apresentar número de indivíduos próximo a 30.

Os dados relativos às variáveis $\mathrm{pH}, \mathrm{Al}, \mathrm{Ca}, \mathrm{Mg}, \mathrm{H}, \mathrm{P}$, $\mathrm{K}$, $\mathrm{C}$, areia, silte, argila, soma de bases $(\mathrm{S}=\mathrm{Ca}+\mathrm{Mg}+$ $\mathrm{Na}+\mathrm{K})$, capacidade de troca catiônica $(\mathrm{CTC}=\mathrm{S}+\mathrm{H}+$ $\mathrm{Al})$, saturação por bases $(\mathrm{V}=100 . \mathrm{S} / \mathrm{T})$ e saturação por alumínio $[\mathrm{m}=100 . \mathrm{Al} /(\mathrm{S}+\mathrm{Al})]$ foram submetidos ao teste de normalidade de Shapiro-Wilk (SAS Institute, 1999) e considerados como atributos das plantas presentes. Em seguida, determinou-se a média de cada uma das variáveis, por espécie, considerando-a como atributo da espécie e associando-a ao número de plantas, para fins das seguintes análises multivariadas: análise de clusters (grupos), pelo método da variância mínima, aferida pela análise combinada a posteriori, e análise discriminante canônica. Após a obtenção dos grupos, seguiram-se as análises de variância multi e univariada e o teste de Tukey, para grupos e para cada uma das variáveis consideradas, respectivamente.

As análises estatísticas foram efetuadas pelo Sistema SAS (Statistical Analysis System), versão 8.2 (SAS Institute, 1999).

\section{Resultados e Discussão}

O estudo foi limitado por um aspecto de amostragem da área experimental, que, originalmente, foi delineada com o objetivo de avaliar impactos da extração de madeira em tora numa área selecionada de 100 ha (Synnott, 1979). Portanto, os dados coletados representam a fração florestal amostrada, sendo possível que a avaliação de outro ponto, ou uma superfície diferente, revele agrupamentos distintos considerando-se a ocorrência de outras espécies, diferentes tamanhos de populações comuns às encontradas neste estudo e outras variações de solo. Essa limitação, contudo, não impediu que se atingisse o objetivo do estudo, porque a quantidade de subparcelas (240) e de árvores das 32 espécies utilizadas (3.556) favoreceram a precisão biométrica e per- mitiram identificar as altas probabilidades de ajuste das espécies em cada grupo respectivo (Tabela 2).

As variáveis de solo estudadas apresentaram distribuição normal, com amplitude de variação do valor W do teste Shapiro-Wilk entre 0,84 e 0,99. Os valores das variáveis de solo são atribuídas às espécies e aos grupos com o propósito de simplificar a terminologia utilizada, mas referem-se às características do solo ocupado pelas populações estudadas.

As espécies foram agrupadas em três grupos ( $\mathrm{Fi}-$ gura 1), definidos pelas altas probabilidades de ajuste $(\operatorname{Pr} \mathrm{j} \mid \mathrm{X})$ das espécies aos respectivos grupos designados (Tabela 2) e estimadas por análise combinada a posteriori.

De acordo com a matriz de correlações, as variáveis mais importantes na definição dos grupos, pela variável canônica 1, foram, respectivamente, $\mathrm{Ca}, \mathrm{Mg}$ e soma de bases (Tabela 3). Duas outras variáveis, definidas pela mesma canônica 1 e também de importância, são saturação por bases, cuja correlação é positiva porém mais baixa, e saturação por alumínio, que apresenta correlação mais alta porém negativa. Já a variável canônica 2, em cuja projeção se diferencia melhor o grupo 3 dos demais (Figura 1), as variáveis mais importantes foram $\mathrm{H}, \mathrm{CTC}, \mathrm{Ke} \mathrm{P}$, com correlações positivas, além de silte e $\mathrm{C}$, cujas correlações são negativas.

A amplitude de variação das principais variáveis de solo, entre as responsáveis pela diferenciação dos grupos demonstra predominância de subparcelas com indicadores de baixa fertilidade (Figuras 2 e 3 ). Esse aspecto foi observado com a distribuição de parcelas em relação ao Ca (Figura 2), tendo-se obtido, no diagrama correspondente, a tendência decrescente de freqüências, com o aumento dos valores do elemento, embora o teste de normalidade tenha indicado a distribuição normal ( $\mathrm{W}=0,84)$.

A análise de variância multivariada detectou diferença altamente significativa entre os grupos com uma probabilidade $(\operatorname{Pr}>\mathrm{F})$ de 0,0001. Esse resultado é

Tabela 1. Características químicas e físicas do solo do Camaipi. Mazagão, AP, 1997.

\begin{tabular}{|c|c|c|c|c|c|c|c|c|c|c|c|c|c|c|c|}
\hline \multirow[t]{2}{*}{ Parâmetros } & \multirow[t]{2}{*}{$\mathrm{pH}$} & $\mathrm{Al}$ & $\mathrm{H}$ & $\mathrm{Ca}$ & $\mathrm{Mg}$ & K & $\mathrm{P}$ & $\mathrm{C}$ & Areia & Silte & Argila & $S$ & CTC & $\mathrm{V}$ & $\mathrm{m}$ \\
\hline & & --- & $--\cdot$ & $\mathrm{nol}_{\mathrm{c}}$ & ) ----- & ------ & \multicolumn{2}{|c|}{$---\left(\mathrm{g} \mathrm{dm}^{-3}\right)---$} & ---- & $\mathrm{g} \mathrm{kg}$ & - & \multicolumn{2}{|c|}{$\left(\mathrm{mmol}_{\mathrm{c}} \mathrm{dm}^{-3}\right)$} & \multicolumn{2}{|c|}{----- (\%) ----- } \\
\hline Média & 4,2 & 18,2 & 60,2 & 2,3 & 2,0 & 0,45 & 1,5 & 26,0 & 205 & 291 & 504 & 0,47 & 8,3 & 5,8 & 80 \\
\hline Desvio-padrão & 0,1 & 2,7 & 16,0 & 0,8 & 0,6 & 0,28 & 0,8 & 4,5 & 90 & 68 & 66 & 0,15 & 1,9 & 1,9 & 4 \\
\hline
\end{tabular}


claro na comparação de médias, por variável (Tabela 4), que sinaliza as diferenças entre grupos de espécies.

Em concordância com os resultados da matriz de correlações (Tabela 3), a comparação de médias (Tabela 4) revelou a diferenciação do grupo 1 dos outros dois. As médias de $\mathrm{Ca}, \mathrm{Mg}$ e soma de bases foram significativamente menores no grupo 1 do que nos demais, ao contrário da média da saturação por alumínio, mais alta. Da mesma forma, a diferenciação do grupo 3 , segundo a variável canônica 2 (Tabela 3), tem suporte na comparação de médias (Tabela 4), com resultados significativamente superiores em H, K, P e CTC em relação aos demais grupos, e inferiores quanto ao silte.

Os grupos 3 e 1 foram os que apresentaram maior número de diferenças entre si, sendo estatisticamente semelhantes apenas em $\mathrm{C}$ e saturação por bases,

Tabela 2. Classificação de espécies arbóreas por família, grupo (Gru), probabilidade de ajuste no grupo designado $(\operatorname{Pr}[\mathrm{j} \mid \mathrm{X}])$, número de indivíduos (Npl), número de subparcelas (Nsubp) e parcelas (Nparc) em que aparecem na Floresta do Camaipi. Mazagão, AP, 1997.

\begin{tabular}{|c|c|c|c|c|c|c|}
\hline Espécie & Família & Gru & $\operatorname{Pr}(\mathrm{j} \mid \mathrm{X})$ & $\mathrm{Npl}$ & Nsubp & Nparc \\
\hline Aspidosperma macrocarpon & Apocynaceae & 1 & 1 & 42 & 34 & 10 \\
\hline Protium opacum & Burseraceae & 1 & 1 & 35 & 34 & 9 \\
\hline Trattinickia rhoifolia & Burseraceae & 1 & 0,99 & 63 & 53 & 12 \\
\hline Sclerolobium guianensis & Cesalpinaceae & 1 & 0,99 & 181 & 72 & 11 \\
\hline Licania sp. & Chrysobalanaceae & 1 & 0,99 & 88 & 63 & 11 \\
\hline Aniba guianensis & Lauraceae & 1 & 1 & 33 & 30 & 11 \\
\hline Lecythis persistens & Lecythidaceae & 1 & 1 & 70 & 42 & 4 \\
\hline Rinorea macrocarpa & Melastomataceae & 1 & 1 & 45 & 43 & 11 \\
\hline Mouriri torquata & Melastomataceae & 1 & 0,99 & 81 & 63 & 12 \\
\hline Miconia prasina & Melastomataceae & 1 & 0,99 & 160 & 153 & 11 \\
\hline Pithecelobium racemosum & Mimosaceae & 1 & 0,94 & 52 & 47 & 11 \\
\hline Pouteria guianensis & Sapotaceae & 1 & 0,90 & 134 & 105 & 11 \\
\hline Vochysia guianensis & Vochysiaceae & 1 & 1 & 79 & 64 & 12 \\
\hline Média & & & & 81,8 & 61,8 & 10,5 \\
\hline Annona sericea & Annonaceae & 2 & 0,99 & 63 & 51 & 11 \\
\hline Geissospermum argenteum & Apocynaceae & 2 & 0,97 & 172 & 120 & 12 \\
\hline Protium pallidum & Burseraceae & 2 & 0,99 & 179 & 122 & 12 \\
\hline Tachigalia myrmecophylla & Cesalpinaceae & 2 & 1 & 188 & 120 & 12 \\
\hline Licania incana & Chrysobalanaceae & 2 & 1 & 274 & 146 & 12 \\
\hline Licania heteromorpha & Chrysobalanaceae & 2 & 0,99 & 266 & 132 & 12 \\
\hline Espécie não identificada & Euphorbiaceae & 2 & 1 & 69 & 47 & 12 \\
\hline Ocotea baturitensis & Lauraceae & 2 & 0,99 & 109 & 73 & 11 \\
\hline Inga edulis & Mimosaceae & 2 & 0,99 & 111 & 30 & 12 \\
\hline Eugenia prosoneura & Myrtaceae & 2 & 0,97 & 34 & 30 & 5 \\
\hline Taralea oppositifolia & Papilionaceae & 2 & 0,99 & 43 & 37 & 10 \\
\hline Manilkara paraensis & Sapotaceae & 2 & 0,90 & 76 & 69 & 12 \\
\hline Rinorea flavescensis & Violaceae & 2 & 0,98 & 76 & 50 & 8 \\
\hline Média & & & & 128 & 79,0 & 10,8 \\
\hline Annona sp. & Annonaceae & 3 & 0,99 & 99 & 62 & 10 \\
\hline Aspidosperma rigidum & Apocynaceae & 3 & 0,96 & 27 & 27 & 12 \\
\hline Holopyxidium jarana & Lecythidaceae & 3 & 1 & 36 & 33 & 12 \\
\hline Inga paraensis & Mimosaceae & 3 & 0,99 & 44 & 36 & 11 \\
\hline Myrcia bracteata & Myrtaceae & 3 & 0,99 & 52 & 41 & 11 \\
\hline Sterculia pilosa & Sterculiaceae & 3 & 1 & 42 & 34 & 8 \\
\hline Média & & & & 50 & 38,8 & 10,7 \\
\hline Total (espécies listadas) & & & & 3.232 & & \\
\hline Total (todas as espécies) & & & & 3.556 & & \\
\hline
\end{tabular}




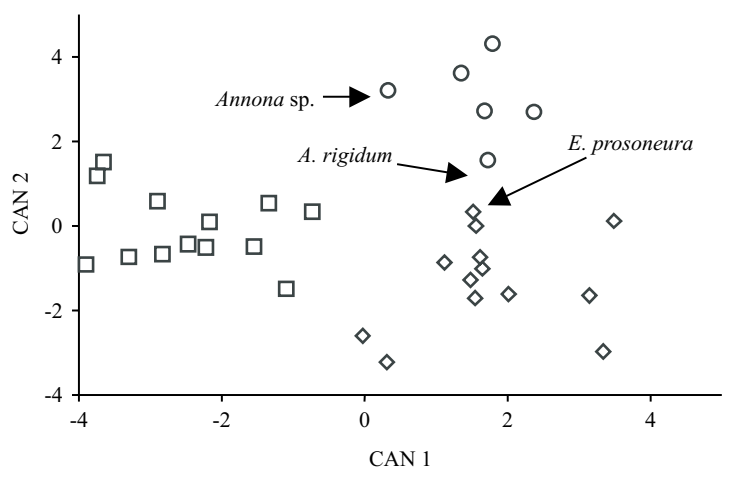

Figura 1. Variáveis canônicas 1 e 2 (CAN 1 e CAN 2) e grupos 1(口), 2(১) e 3(o) formados com as espécies arbóreas da Floresta do Camaipi. Mazagão, AP, 1997.

Tabela 3. Correlações parciais entre variáveis originais e canônicas 1 e 2 (CAN 1 e CAN 2) de espécies arbóreas da Floresta do Camaipi. Mazagão, AP, $1997^{(1)}$.

\begin{tabular}{lcc}
\hline Variáveis originais & \multicolumn{2}{c}{ Correlações } \\
\cline { 2 - 3 } & CAN 1 & CAN 2 \\
& $\mathrm{R}^{2}=0,82$ & $\mathrm{R}^{2}=0,73$ \\
\hline Número de plantas & 0,27 & $-0,50$ \\
$\mathrm{pH}$ & 0,34 & 0,29 \\
$\mathrm{Al}$ & 0,34 & 0,44 \\
$\mathrm{Ca}$ & 0,91 & $-0,17$ \\
$\mathrm{Mg}$ & 0,82 & 0,15 \\
$\mathrm{H}$ & 0,37 & 0,80 \\
$\mathrm{P}$ & 0,44 & 0,68 \\
$\mathrm{~K}$ & 0,46 & 0,77 \\
$\mathrm{C}$ & 0,02 & $-0,74$ \\
Areia & 0,16 & 0,63 \\
Silte & $-0,11$ & $-0,78$ \\
Argila & $-0,07$ & 0,18 \\
Soma de bases & 0,90 & 0,19 \\
CTC & 0,42 & 0,77 \\
Saturação por bases & 0,66 & $-0,54$ \\
Saturação por Al & $-0,78$ & $-0,09$ \\
\hline
\end{tabular}

(1)As variáveis canônicas são combinações lineares das variáveis quantitativas; a variável canônica 1 representa a combinação linear de máxima correlação múltipla possível com os grupos; a canônica 2 representa a de máxima correlação possível com os grupos, não correlacionada com a canônica 1

além de pH e argila, que mostraram-se iguais nos três grupos (Tabela 4). Esses dois grupos representaram as tendências mais distantes (Figura 1), em termos de variáveis de solo, contudo não foram diferentes do ponto de vista estatístico quanto ao número médio de plantas (Tabela 4). O grupo 2, que tendeu a ocupar a faixa intermediária das variáveis de solo, apresentou praticamente a mesma quantidade de semelhanças e dessemelhanças em relação aos demais grupos.

Depreende-se que a maior presença, estatisticamente detectada, de um grupo de espécies que ocupa a faixa de valores médios entre os indicadores de fertilidade do solo com relação a outro provavelmente não se deva ao acaso. Os grupos 1 e 2 foram semelhantes quanto ao número médio de plantas, o que indica a capacidade de ocupação de sítio; quanto ao P, que, segundo Vitousek (1984) e Silver (1994) é a principal limitação nutricional da floresta tropical; e quanto ao $\mathrm{Ca}$ e $\mathrm{Mg}$, nutrientes essenciais. Além disso, a maior abundância das populações do grupo 2 foi detectada somente com relação ao grupo 3, identificado com valores superiores entre os indicadores da fertilidade do solo local. A partir desses dados pode-se inferir que as espécies arbóreas estudadas apresentaram-se em grupos com características comuns de adaptação às condições e recursos do solo que, possivelmente, influíram no tamanho de suas populações, no Camaipi.

Entre as famílias botânicas constituídas por mais de uma espécie, Melastomataceae ficou limitada a um único grupo (Tabela 2). Todas as demais, mesmo com apenas duas espécies, tiveram representantes em mais de um grupo, podendo-se notar, ainda, que outras duas famílias (Mimosaceae e Apocynaceae), constituídas por três espécies, foram representadas nos três grupos.

Entre as espécies destacam-se os resultados obtidos com Eugenia prosoneura que, com apenas 34 indivíduos, está incluída no grupo 2 , repleto de espécies de alta densidade populacional. No grupo 3, encontra-se Annona sp., com 99 indivíduos e mais próxima do grupo 1, tendendo ao outro extremo dos indicadores de fertilidade (Figura 1). Aspidosperma rigidum está mais bem representada no grupo 3, o que parece apropriado em razão de sua pequena população (27 indivíduos), contudo desse grupo é a espécie mais próxima do grupo 2.

O peso ecológico das 32 espécies selecionadas deve ser determinante na formação qualitativa e quantitativa da serapilheira local, com influência relativamente difícil de isolar (Vitousek \& Sanford Junior, 1986), mas expressiva porque representa aproximadamente $82 \%$ das árvores dessa amostra do Camaipi (Tabela 2) e apenas $15 \%$ das espécies locais, considerando que Mori et al. (1989), nesse sítio, encontraram 205 espécies. 

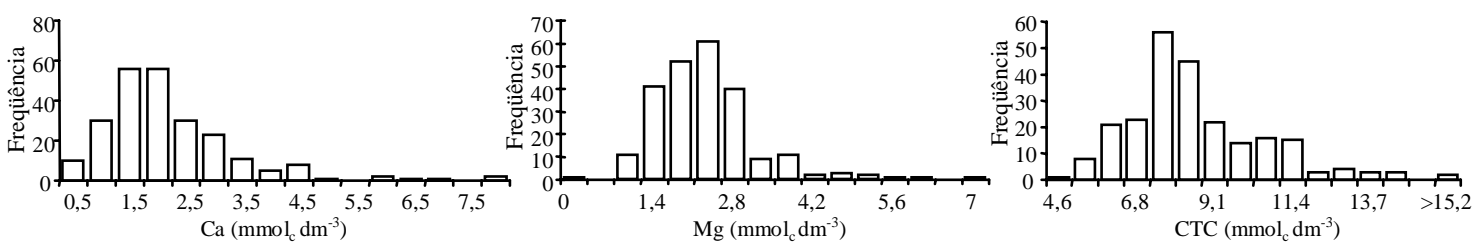

Figura 2. Histogramas com a distribuição de freqüências de subparcelas em relação a valores de Ca, $\mathrm{Mg}$ e CTC no solo da Floresta do Camaipi. Mazagão, AP, 1997.
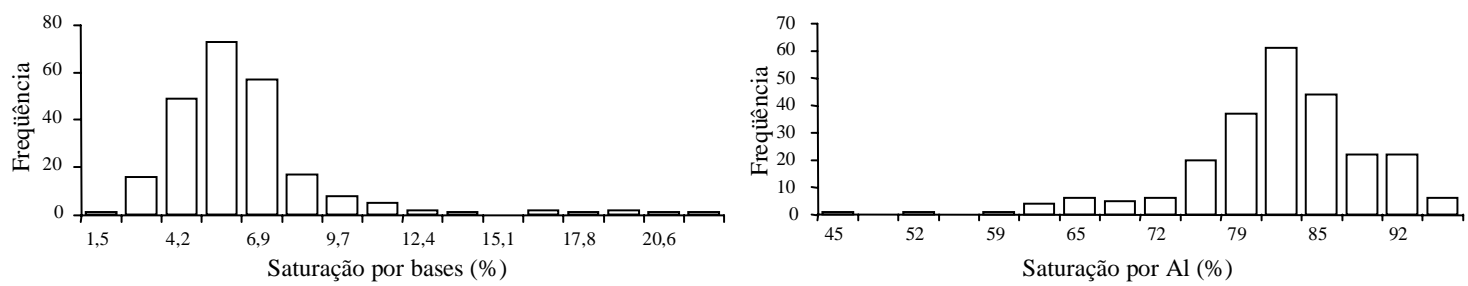

Figura 3. Histogramas com a distribuição de freqüências de subparcelas em relação a valores porcentuais de saturação por bases e saturação por alumínio no solo da Floresta do Camaipi. Mazagão, AP, 1997.

Tabela 4. Comparação entre os grupos 1, 2 e 3 quanto ao número médio de plantas de espécies arbóreas (NP) e variáveis de solo na Floresta do Camaipi. Mazagão, AP, 1997(1).

\begin{tabular}{ccccccccc}
\hline Grupo & $\mathrm{NP}$ & $\mathrm{pH}$ & $\mathrm{Al}$ & $\mathrm{Ca}$ & $\begin{array}{c}\mathrm{Mg} \\
\left(\mathrm{mmol}_{\mathrm{c}} \mathrm{dm}^{-3}\right)\end{array}$ & $\mathrm{H}$ & $\mathrm{K}$ & $\begin{array}{c}\mathrm{P} \\
\left(\mathrm{mg} \mathrm{dm}^{-3}\right)\end{array}$ \\
\hline 1 & $73 \mathrm{ab}$ & $4,1 \mathrm{a}$ & $17,2 \mathrm{~b}$ & $1,9 \mathrm{~b}$ & $1,7 \mathrm{~b}$ & $52,3 \mathrm{~b}$ & $0,3 \mathrm{~b}$ & $1,1 \mathrm{~b}$ \\
2 & $128 \mathrm{a}$ & $4,1 \mathrm{a}$ & $17,4 \mathrm{ab}$ & $2,2 \mathrm{a}$ & $1,9 \mathrm{a}$ & $53,0 \mathrm{~b}$ & $0,3 \mathrm{~b}$ & $1,2 \mathrm{~b}$ \\
3 & $50 \mathrm{~b}$ & $4,2 \mathrm{a}$ & $18,1 \mathrm{a}$ & $2,1 \mathrm{a}$ & $2,0 \mathrm{a}$ & $62,3 \mathrm{a}$ & $0,5 \mathrm{a}$ & $1,6 \mathrm{a}$ \\
\hline & $\mathrm{C}$ & Areia & Silte & Argila & $\mathrm{S}$ & $\mathrm{CTC}$ & $\mathrm{V}$ & $\mathrm{m}$ \\
\hline 1 & $\left(\mathrm{mg} \mathrm{dm}^{-3}\right)$ & $--------------\left(\mathrm{g} \mathrm{kg}^{-1}\right)$ & ------------ & ---- & $\left(\mathrm{mmol}_{\mathrm{c}} \mathrm{dm}^{-3}\right)----$ & $-------(\%)$ & ------- \\
\hline & $25,3 \mathrm{~b}$ & $200 \mathrm{~b}$ & $303 \mathrm{a}$ & $497 \mathrm{a}$ & $0,39 \mathrm{~b}$ & $7,3 \mathrm{~b}$ & $5,4 \mathrm{~b}$ & $82 \mathrm{a}$ \\
3 & $26,0 \mathrm{a}$ & $197 \mathrm{~b}$ & $310 \mathrm{a}$ & $493 \mathrm{a}$ & $0,45 \mathrm{a}$ & $7,5 \mathrm{~b}$ & $6,1 \mathrm{a}$ & $80 \mathrm{~b}$ \\
\hline
\end{tabular}

${ }^{(1)}$ Grupos seguidos de mesma letra, nas colunas, não diferem entre si pelo teste de Tukey a 5\% de probabilidade.

Debski et al. (2000) sugerem a existência de gradientes de solo além de mecanismos denso-dependentes relacionados com as abundâncias de diversas espécies arbóreas em uma floresta tropical úmida da Austrália. Assim, a abundância das espécies pode refletir a adaptação às condições nutricionais locais. Em condições edáficas, como as do Camaipi, podem ser importantes a exploração profunda do solo em razão do período seco prolongado (Sternberg et al., 1998), a eficiência em absorver nutrientes, especialmente P, a tolerância ao Al (Vitousek \& Sanford Junior, 1986) e a associação simbiótica com micorrizas (Siqueira et al., 1998).

Ainda que não tenham sido encontradas evidências do maior peso de fatores determinísticos, em relação ao acaso, nos termos mencionados por Brokaw \& Busing (2000), os resultados sugerem que as espécies mais abundantes da Floresta do Camaipi são agrupáveis por características de solo e que há uma relação multivariada entre características edáficas e as relativas abundâncias de um grupo ponderável das espécies arbóreas estudadas.

\section{Conclusões}

1. As espécies formam três grupos e as características de solo mais importantes na formação desses grupos são $\mathrm{Ca}, \mathrm{Mg}, \mathrm{K}$ e alumínio.

2. A concentração das espécies da família Melastomataceae no grupo 1, de menor fertilidade do solo, sugere a estratégia oligotrófica, em relação à família botânica. 


\section{Agradecimentos}

Ao Dr. João Olegário Pereira de Carvalho, Embrapa-Centro de Pesquisa Agroflorestal da Amazônia Oriental, pela orientação técnica na condução das pesquisas na Floresta do Camaipi; a Sandoval de Jesus Amaral, operário rural da Embrapa-Centro de Pesquisa Agroflorestal do Amapá, pela identificação das espécies inventariadas.

\section{Referências}

BEGON, M.; HARPER, J. L.; TOWSEND, C. R. Ecology: individuals, populations and communities. Oxford: Blackwell, 1986. 876 p

BROKAW, N.; BUSING, R. T. Niche versus chance and tree diversity in forest gaps. Trends in Ecology and Evolution, Oxford, v. 15, n. 5, p. 183-188, 2000.

CONDIT, R.; HUBBELL, S.; LAFRANKIE, J.; SUKUMAR, R.; MANOKARAN, N.; FOSTER, R. B.; ASHTON, P. S. Species-area and species-individual relationships for tropical trees: a comparison of three 50-ha plots. Journal of Ecology, Oxford, v. 84, p. 549$562,1996$.

CONNEL, J. H.; GREEN, P. T. Seedlings dynamics over thirty-two years in a tropical rain forest tree. Ecology, Durham, v. 81 , n. 2 , p. $568-584,2000$

DEBSKI, I.; BURSLEM, D. F. R. P.; LAMB, D. Ecological processes maintaining differential tree species distributions in an Australian subtropical rain forest: implications for models of species coexistence. Journal of Tropical Ecology, Cambridge, Inglaterra, v. 16, p. 387-415, 2000.

EMBRAPA. Centro Nacional de Pesquisa de Solos (Rio de Janeiro, RJ). Sistema brasileiro de classificação de solos. Brasília: Embrapa-SPI/Embrapa-CNPS, 1999. 412 p.

HARMS, K. E.; WRIGHT, S. J.; CALDERÓN, O.; HERNÁNDEZ, A.; HERRE, E. A. Pervasive densitydependent recruitment enhances seedling diversity in a tropical forest. Nature, London, v. 4, p. 493-495, 2000.

HUSTON, M. Soil nutrients and tree species richness in Costa Rican forests. Journal of Biogeography, Oxford, v. 7, p. 147-157, 1980.

IBGE. Diretoria de Geociências (Rio de Janeiro, RJ). Manual técnico da vegetação brasileira. Rio de Janeiro, $1992.92 \mathrm{p}$.
JORDAN, C. F. Nutrient cycling processes and tropical forest management. In: GÓMEZ-POMPA, A.; WHITMORE, T. C.; HADLEY, M. (Ed.). Rain forest regeneration and management. Paris: Unesco, 1991. p. 159-180. (Man and the Biosphere Series, 6).

JORDAN, H.; HERRERA, R. Tropical rain forests: are nutrients really critical? American Naturalist, Chicago, v. 117, n. 2, p. 167-180, 1981.

LAMPRECHT, H. Silvicultura en los trópicos. Gottingen: Instituto de Silvicultura de la Universidad de Gottingen, 1986. 335 p.

MARKEWITZ, D.; DAVIDSON, E. A.; FIGUEIREDO, R. O.; VICTORIA, R. L.; KRUSCHE, A. V. Control of cation concentrations in stream waters by surface soil processes in an Amazonian watershed. Nature, London, v. 410 , p. $802-805,2001$

MEGGER, B. J. Archeological evidence for the impact of mega-niño events on Amazonia during the past two millennia. Climatic Change, Dordrecht, v. 28, n. 4, p. 321$338,1994$.

MORI, S. A.; RABELO, B. V.; TSOU, C.; DALY, D. Composition and structure of an Eastern Amazonian forest at Camaipi, Amapá, Brazil. Boletim do Museu Paraense Emilio Goeldi, Série Botânica, Belém, v. 5, n. 1, p. 13$18,1989$.

OLSON, R. A.; CLARK, R. B.; BERNETT, J. H. The enhancement of soil fertility by plant roots. American Scientist, New Haven, v. 69, p. 378-384, 1981.

RABELO, B. V. (Coord.). Zoneamento ecológico econômico do Amapá: ciclo de seminários do zoneamento ecológico econômico do Estado do Amapá: caderno síntese de resultados. [Brasília]: Ministério do Meio Ambiente dos Recursos Hídricos e da Amazônia Legal/Secretaria de Estado de Meio Ambiente, Ciência e Tecnologia do Amapá, 1999. 74 p. Projeto de Gestão Ambiental Integrado: área prioritária, 1 .

REBELE, F. Competition and coexistence of rhizomatous perennial plants along a nutrient gradient. Plant Ecology, Dordrecht, v. 147, p. 77-94, 2000.

RODRIGUES, T. E. Solos da Amazônia. In: ALVAREZ VENEGAS, H.; FONTES, L. E. F. O solo nos grandes domínios morfoclimáticos do Brasil e o desenvolvimento sustentado. Viçosa, MG: Sociedade Brasileira de Ciência do Solo/UFV, 1996. p. 19-60.

SAS INSTITUTE (Cary, Estados Unidos). SAS OnlineDoc: version 8. Cary, 1999. 1 CD-ROM. 
SILVER, W. L. Is nutrient availability related to plant nutrient use in humid tropical forests? Oecologia, Berlin, v. 98 , p. 336-343, 1994.

SINGH, J. S.; RAGHUBANSHI, A. S.; SINGH, R. S.; SRIVASTAVA, S. C. Microbial biomass acts as a source of plant nutrients in dry tropical forest and savanna. Nature, London, v. 338, p. 499-500, 1989.

SIQUEIRA, J. O.; CARNEIRO, M. A. C.; CURI, N. C.; ROSADO, S. C. S.; DAVIDE, A. C. Mycorrhizal colonization and mycotrophic growth of native woody species as related to successional groups in Southeastern Brazil. Forest Ecology and Management, Amsterdam, v. 107, p. 241-252, 1998.

STERNBERG, C. S. C.; GREEN, L.; MOREIRA, M. Z.; NEPSTAD, D.; MARTINELLI, L. A.; VICTORIA, R. Root distribution in an Amazonian seasonal forest as derived from $\delta^{13} \mathrm{C}$ profiles. Plant and Soil, The Hague, v. 205, p. 45-50, 1998.

SYNNOTT, T. J. A manual of permanent plot procedures for tropical rainforests. Oxford: Commonwealth Forestry Institute, 1979. 67 p. (Occasional Paper, 14).

VELOSO, H. P.; RANGEL FILHO, A. L. R.; LIMA, J. C. A. Classificação da vegetação brasileira, adaptada a um sistema universal. Rio de Janeiro: IBGE, 1991. 123 p.

VITOUSEK, P. M. Litterfall, nutrient cycling, and nutrient limitation in tropical forests. Ecology, Durham, v. 65, n. 1, p. 285-298, 1984.

VITOUSEK, P. M.; SANFORD JUNIOR, R. L. Nutrient cycling in moist tropical forest. Annual Review on Ecology Systematics, Palo Alto, v. 17, p. 137-167, 1986. WHITMORE, T. C. An introduction to tropical rain forests. Oxford: Clarendon, 1990. 226 p. 